\title{
Lait et fromage dans un pays andin : quelle place pour les filières artisanales péruviennes face aux industries laitières?
}

\author{
C. Aubron ${ }^{1}$
}

\section{Mots-clés}

Elevage - Lait - Fromage - Secteur informel - Industrie laitière - Politique agricole - Région andine - Pérou.

\begin{abstract}
Résumé
Depuis le début des années 1990, la production laitière péruvienne est en forte croissance. Le développement des filières laitières répond à celui d'un marché protégé des importations, en expansion du fait de la croissance démographique urbaine et rendu accessible par l'amélioration du réseau routier. II concerne les filières artisanale et industrielle qui sont liées en amont et en aval par des relations d'interdépendance et des rapports de force. A partir d'enquêtes technico-économiques auprès des éleveurs laitiers de différentes régions, cet article montre que ce développement laitier se traduit par d'importantes inégalités de revenus entre les catégories de producteurs, reflet de leur accès différencié aux ressources. Les données statistiques consultées et les entretiens réalisés avec différents acteurs des filières laitières péruviennes permettent d'évaluer les enjeux et les limites des démarches qualité mises en œuvre dans les filières artisanales pour faire face aux industries. L'article s'interroge finalement sur l'impact des accords de libre-échange dans lesquels le Pérou est engagé sur les filières laitières nationales et conclut par des propositions de politiques d'accompagnement à ce développement laitier.
\end{abstract}

\section{INTRODUCTION}

Depuis 1990, la production laitière péruvienne est en forte croissance dans les Andes comme sur la côte Pacifique, et dans une moindre mesure dans le piémont amazonien. Dans un pays marqué dans le même temps par l'avènement de politiques néolibérales centrées sur la promotion des productions d'exportation (mangue, café, asperge, paprika, artichaut), ce développement laitier tourné vers le marché intérieur passe relativement inaperçu. Adoptée par de nombreuses familles paysannes des Andes, la production laitière s'intègre pourtant avec succès dans leurs systèmes de production et joue un rôle-clé pour leur sécurité alimentaire qu'elles tentent de garantir par la vente régulière de lait ou de fromage (3). Dans ce pays qui présente l'un des plus faibles niveaux de consommation de produits laitiers d'Amérique du Sud, ce manque de lisibilité de la dynamique laitière constitue un obstacle à la définition de

1. Montpellier SupAgro, UMR Elevage des ruminants en régions chaudes, 2 place Pierre Viala, 34060 Montpellier Cedex 1, France.

Tél. : +33 (0)4 99612265 ; fax : +33 (0)4 67545694

E-mail : aubron@supagro.inra.fr politiques ou de projets adaptés. Il est encore plus marqué concernant les filières artisanales qui absorberaient, d'après les estimations du ministère de l'Agriculture, la moitié de la production nationale, l'autre moitié étant destinée à la filière industrielle. Au Pérou, comme dans d'autres pays en développement (16), la filière laitière artisanale est en effet considérée comme un fourre-tout regroupant diverses activités de production, de transformation et de commercialisation jugées informelles, dispersées et peu productives et dont le potentiel de développement est méconnu.

Cherchant à combler ces insuffisances, cet article analyse les dynamiques en cours dans les filières laitières péruviennes, les relations d'interdépendance qui les unissent et la différenciation des systèmes de production laitiers qui les approvisionnent. Il explique en quoi le marché péruvien des produits laitiers constitue aujourd'hui un marché porteur qui permet de comprendre la croissance de la production nationale. Il discute ensuite des inégalités générées par ce développement laitier, de l'opportunité de la mise en place de démarches qualité dans les filières fromagères artisanales et de l'impact des accords de libre-échange dans lesquels le Pérou est engagé sur les filières laitières nationales, avant de conclure par des propositions de politiques d'accompagnement. 


\section{MATERIEL ET METHODES}

Les filières laitières péruviennes, leur fonctionnement actuel et les transformations qu'elles connaissent constituent l'objet central de cette recherche. D'après Malassis (26), « l'étude de filière comporte deux aspects fondamentaux : son identification (produits, itinéraires, agents, opérations) et l'analyse des mécanismes de sa régulation (structure et fonctionnement des marchés, interventions de l'Etat, planification) ». Selon Ghersi et coll. (18), l'approche filière privilégie " l'étude des phénomènes d'interdépendance et d'intégration qui caractérisent l'appareil de production/transformation/distribution des produits agroalimentaires, ainsi que l'analyse des rapports de force et de domination entre les différents acteurs qui interviennent aux stades successifs du processus ». S'intégrant dans une recherche plus large sur l'évolution des systèmes agraires laitiers andins, cette étude de filière adopte un point de vue qui place les systèmes de production agricoles et les relations de concurrence qu'ils entretiennent au cœur de l'analyse. Pour la très grande majorité des produits agricoles échangés dans le monde, les filières restent en effet entremêlées et interdépendantes au niveau de ce «nœud de la production» (13). Utilisant les méthodes de « l'agriculture comparée » (13), le travail qui suit cherche à explorer ce nœud en étudiant la différenciation des systèmes de production laitiers péruviens et en comparant leur niveau de productivité du travail.

Les données mobilisées dans ce cadre d'analyse sont issues d'un travail de terrain d'une durée de deux ans dans une communauté paysanne du versant occidental des Andes, combinant observations

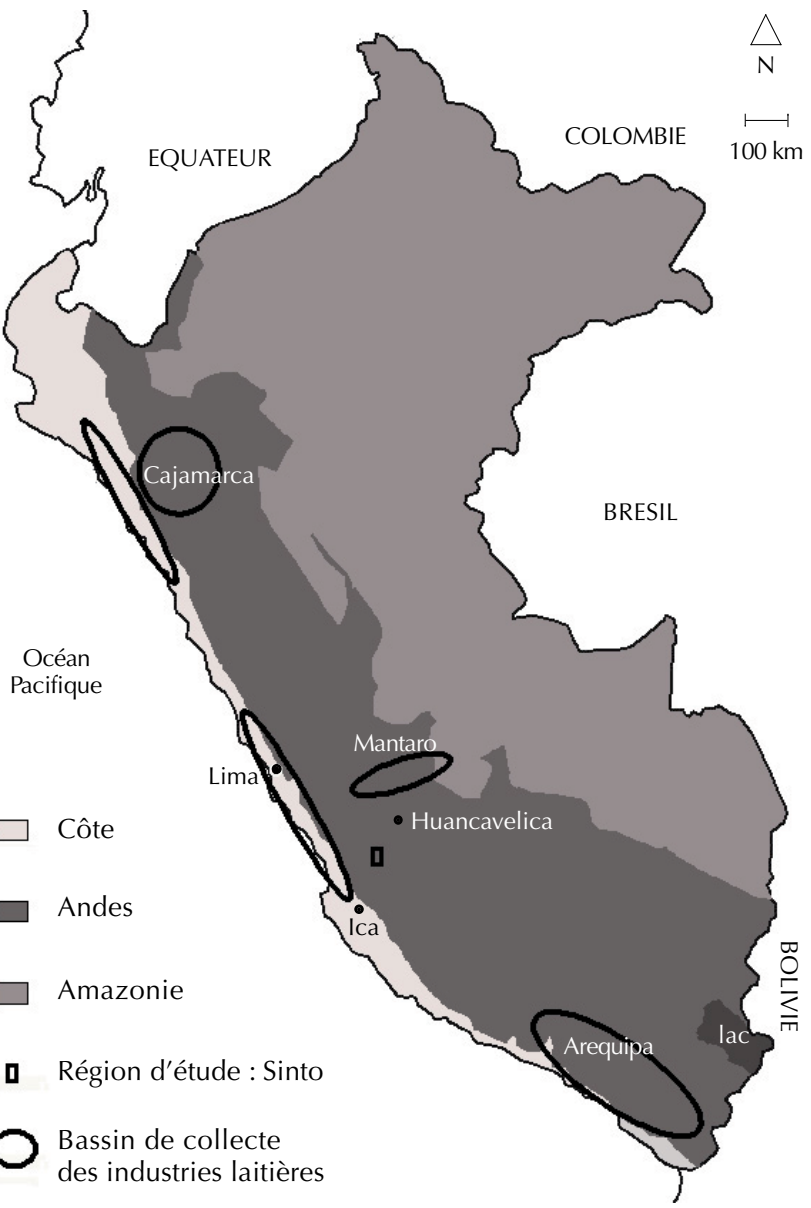

Figure 1 : carte générale du Pérou et positions des bassins laitiers industriels. et enquêtes auprès des éleveurs (50 entretiens, puis suivi mensuel de huit familles pendant un an). Par rapport aux trois principaux bassins laitiers péruviens investis par les industries laitières (régions andines d'Arequipa et de Cajamarca, et côte Pacifique des environs de Lima ; figure 1), la communauté de Sinto constitue un bassin laitier marginal qui alimente deux sous-filières artisanales et dans lequel la production laitière est néanmoins en forte croissance depuis 20 ans. Les résultats de ce travail de terrain, c'est-à-dire la formalisation du fonctionnement et de la dynamique de ce système agraire laitier andin au niveau local, ne seront restitués ici que dans la mesure où ils alimentent l'analyse à l'échelle plus globale des filières laitières nationales.

Cette compréhension de l'amont des filières laitières à Sinto a été complétée par une recherche bibliographique sur huit régions andines et par des enquêtes auprès des éleveurs de quatre bassins laitiers, autorisant une comparaison technico-économique des systèmes de production laitiers. Les informations relatives au fonctionnement aval des filières laitières, à la consommation des produits laitiers et aux politiques laitières proviennent d'un travail bibliographique, d'entretiens auprès des autres acteurs de ces filières (transporteurs, commerçants, industriels, consommateurs) et de la consultation de bases de données du ministère de l'Agriculture, de l'Institut national de l'information et des statistiques (Inei), du service des douanes et de la FAO. Si les volumes et les prix pratiqués par la filière industrielle sont relativement bien connus car comptabilisés par les entreprises, le mutisme quasi total de ces données sur les filières laitières artisanales rend leur analyse parfois délicate. Les coefficients d'équivalent lait utilisés pour sommer les volumes de lait produits ou échangés sont ceux de la FAO, basés sur les taux de matière sèche. Ceux permettant de calculer et de comparer des prix par litre d'équivalent lait correspondent aux rendements du processus de transformation (28).

\section{RESULTATS}

\section{Les filières laitières péruviennes : diversité et interdépendance}

Si elle demande à être étayée, la distinction entre sous-filières industrielle et artisanale est opérationnelle en première analyse dans le cas péruvien : l'artisanat est caractérisé par la maîtrise que le producteur a du processus de travail alors que la filière industrielle mobilise des techniques à haute intensité de capital et fait appel au salariat (1).

L'implantation des industries au Pérou remonte aux années 1940 et concerne des régions andines comme côtières (figure 1). La sousfilière industrielle est caractérisée par une forte concentration : trois entreprises (Gloria, péruvienne, Laive, actuellement contrôlée par des capitaux chiliens, et la multinationale Nestlé) possèdent actuellement plus de 90 p. 100 des parts de marché. Le principal produit laitier industriel au Pérou est le lait évaporé (lait condensé d'un facteur 2), conditionné en boîte de conserve, qui représente 80 p. 100 des ventes en valeur (10). Les industries sont également les premiers importateurs de produits laitiers (figure 2), que ce soit pour les incorporer sous forme de lait en poudre, en mélange avec la matière première nationale, ou pour les vendre directement comme produit fini (fromages, beurres).

Les sous-filières artisanales sont elles-mêmes diverses (figure 2) : dans la filière fermière, les éleveurs laitiers transforment eux-mêmes le lait qu'ils produisent en un fromage peu pressé, moulé à la main ou dans un cylindre de paille tressée et généralement fortement salé qu'ils vendent chaque semaine à des négociants. Dans la filière des fromageries, des unités de transformation artisanales associatives ou privées mobilisant de la main-d'œuvre familiale ou 


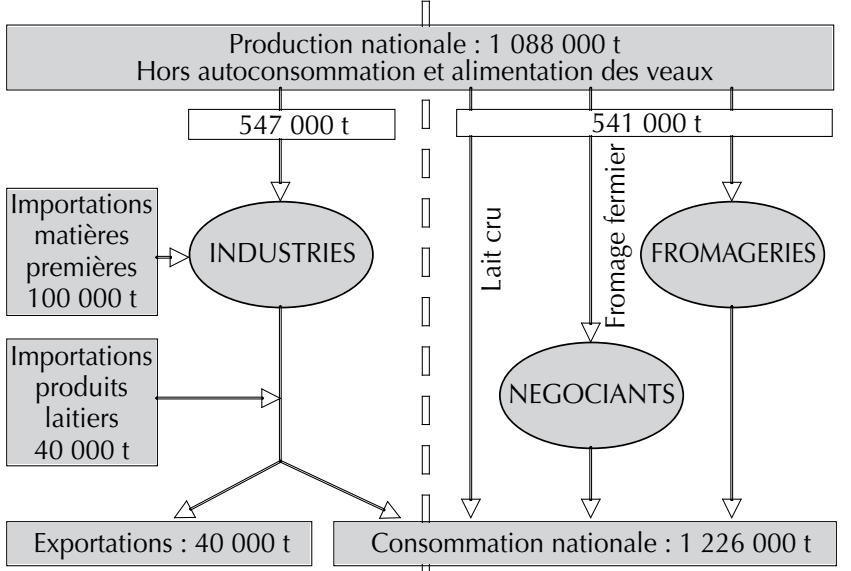

FILIERE INDUSTRIELLE

Figure 2 : schéma d'organisation des filières laitières péruviennes. Les valeurs de volumes correspondent à l'année 2003 (équivalents lait de la FAO) ; Minag, 2003 ; Webb et Fernández Baca, 2004.

salariée (1 à 4 employés) élaborent, à partir du lait qu'elles collectent auprès des producteurs, et commercialisent un fromage qui s'apparente au fromage fermier, mais dont la teneur en sel est moindre et la forme comme la texture plus homogènes. Certaines de ces fromageries produisent également des fromages de maturité plus longue (queso andino, tilsit, tipo suiso), de la mozzarella, du yaourt à boire ou de la confiture de lait. Le queso mantecoso de Cajamarca présente à la fois des attributs de la filière fermière et de la filière des fromageries puisqu'il est élaboré en deux étapes : l'une est entre les mains des éleveurs laitiers qui fabriquent le quesillo et l'autre des fromagers qui l'utilisent ensuite comme matière première (8). Enfin, dans la filière lait cru, les producteurs acheminent leur lait à pied, en transport en commun ou en camionnette et le vendent sur les marchés et dans des échoppes. La majorité des producteurs de lait approvisionnant la filière fermière et la filière des fromageries vivent dans les régions andines. La filière lait cru fonctionne quant à elle aux abords de la plupart des villes, qu'elles soient situées dans les Andes ou sur la côte Pacifique. Les marchés urbains des grandes villes côtières absorbent l'essentiel de la production laitière artisanale et industrielle péruvienne.

Loin de fonctionner isolément, ces différentes filières sont liées par des relations d'interdépendance : dans de nombreux territoires elles sont en concurrence pour leur approvisionnement en matière première laitière, ce qui se traduit par un relatif alignement des prix au producteur quelle que soit la filière qu'il alimente (3). Quand la filière artisanale a le monopole des approvisionnements dans une région, l'industrie qui souhaite s'y établir y parvient en offrant pendant un temps des prix d'achat plus élevés. Elle propose aussi aux fournisseurs des services d'appui technique et surtout de crédit que les fromageries ne sont pas en mesure de fournir (14). Ces rapports de force entre industries et fromageries s'expriment aujourd'hui dans la vallée du Mantaro où l'entreprise Gloria collecte du lait depuis 2005. Si elles ont leurs logiques propres autour de produits qui leur sont plus ou moins spécifiques, les filières industrielle et artisanale sont également concurrentes pour développer leurs parts de marché. C'est ainsi que le fromage artisanal et le lait évaporé entrent dans des proportions variables dans la préparation de plusieurs plats typiques péruviens et que l'évolution du prix au consommateur liménien du fromage est similaire à celle observée pour la boîte de lait évaporé (3). Les négociants se réfèreraient même au prix du lait évaporé au consommateur pour fixer le prix au producteur de fromage fermier (32). Egalement repérée

par d'autres travaux $(16,33)$, cette interdépendance entre sousfilières approvisionnant le marché national des produits laitiers justifie l'intérêt accordé aux dynamiques en cours dans la filière industrielle, essentielles pour comprendre les transformations et les perspectives des filières artisanales.

\section{Un contexte national favorable au développement des filières laitières}

Plusieurs éléments externes aux systèmes de production ou relatifs à l'insertion de l'élevage laitier en leur sein concourent à faire depuis 1990 du marché péruvien des produits laitiers un marché qui peut être qualifié de porteur.

Tout d'abord, le début des années 1990 marque au Pérou une rupture en termes de politique laitière, puisque le pays passe d'une politique de subventions aux importations de lait en poudre à une politique de protection du marché national. Pendant les années 1970 et 1980, le lait en poudre importé destiné au secteur industriel concurrence la production nationale, limitant les volumes collectés au Pérou par les industries et exerçant une pression à la baisse sur les prix au producteur (24). En 1993, de manière paradoxale dans un contexte de libéralisation de l'économie, le gouvernement de Fujimori instaure des taxes à l'importation pour quatre produits identifiés comme sensibles, dont le lait fait partie, sous la forme d'un système dit de frange de prix avec une taxe fixe égale à 25 p. 100 du prix CAF (coûts, assurances, fret : prix du bien à la frontière du pays importateur), complétée par une taxe variable qui s'applique dès lors que le prix FAB (franco à bord : prix du bien embarqué dans le port du pays exportateur) est inférieur à un prix cible redéfini deux fois par an (5, 7). Ces barrières douanières, combinées à la hausse du prix mondial du lait en poudre, suite à l'instauration de la politique des quotas laitiers européens, se traduisent depuis 1993 par une forte croissance de la production nationale qui se substitue aux importations de produits laitiers (figure 3). Ceci contribue à expliquer le relatif maintien du prix réel du lait au producteur péruvien, par rapport à celui d'autres produits agricoles concurrencés par les importations (figure 4). Ce prix attractif du lait constitue pour les paysans péruviens une puissante incitation à la mise en place de l'élevage laitier. Néanmoins, les différents accords de libre-échange signés récemment par le Pérou (intégration de la Communauté andine des nations en 2005, signature d'un traité de libre-échange avec les Etats-Unis en 2005 en attente de ratification par le congrès américain, négociation de l'intégration du Marché commun du Sud, Mercosur) prévoient la suppression des taxes à l'importation de produits laitiers en provenance des pays signataires dans un délai maximum de quinze ans.

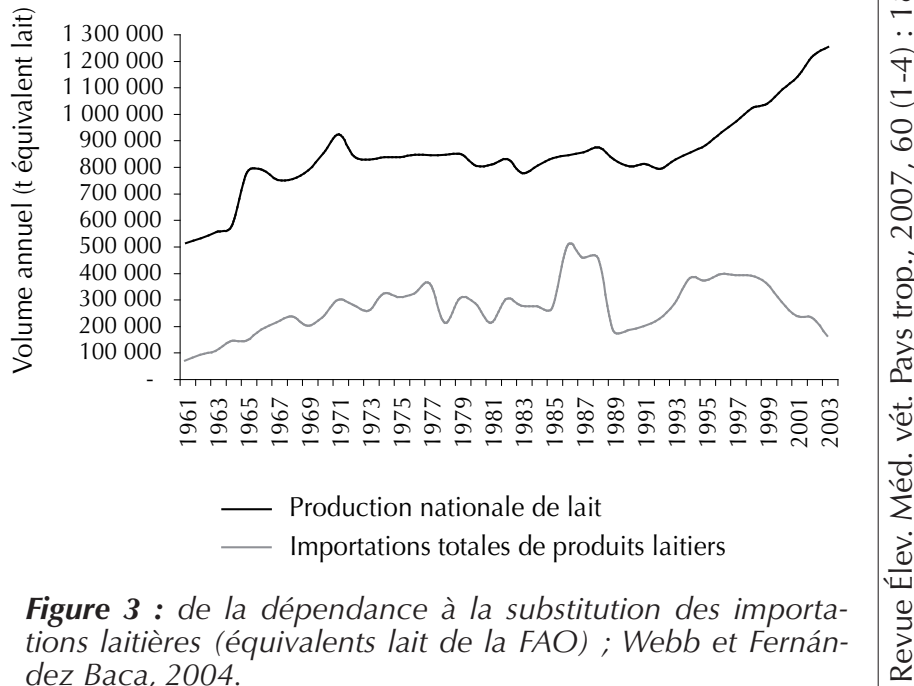




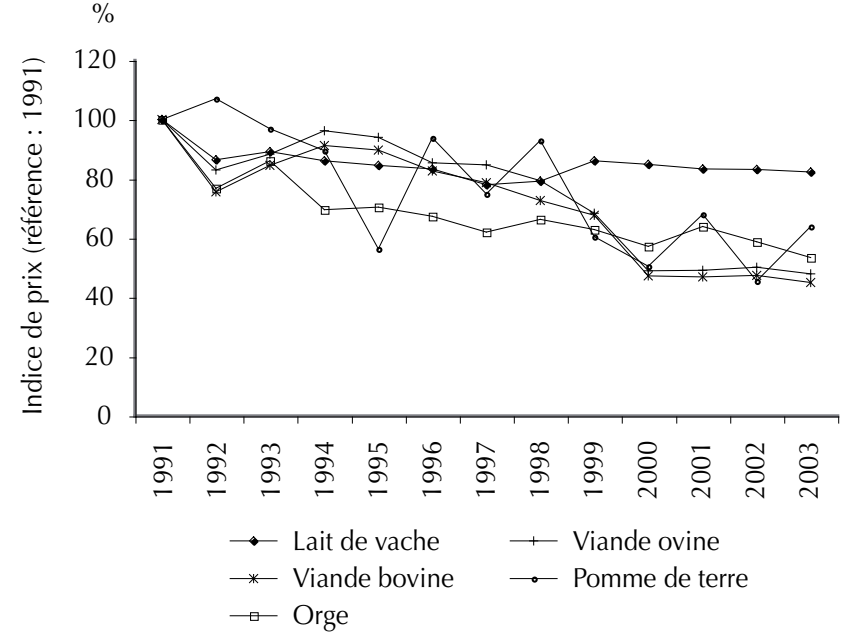

Figure 4 : maintien relatif du prix réel du lait au Pérou, payé au producteur, 1991-2003. Inei, 2001 ; Inei, 2003 ; Inei, 2004 ; Webb et Fernández Baca, 2004.

Avec 50,5 kg d'équivalent lait par habitant et par an, le Pérou présente l'un des plus faibles niveaux moyens de consommation de produits laitiers d'Amérique du Sud. Il n'existe aucune étude approfondie de la consommation péruvienne de produits laitiers mais, à l'image de ce qui est observé dans la Bolivie voisine (17), elle apparaît comme étant très hétérogène et fortement corrélée au niveau de revenus des familles (2). Nombreux sont ainsi les Péruviens pauvres qui ne consomment pas d'autres produits laitiers que ceux distribués par les programmes sociaux, si tant est qu'ils y aient accès. Par ailleurs, la stagnation de la consommation par tête autour de ce faible niveau moyen observée depuis 1990 occulte la très forte expansion du marché en volume liée, d'une part, à la croissance démographique et, d'autre part, à l'urbanisation. Cette dernière tend à changer les modes de consommation (15) vers une plus forte participation des produits laitiers au régime alimentaire (yaourt, petit déjeuner avec du lait, sandwichs au fromage, mozzarella pour pizza, pâtisseries à la confiture de lait, etc.).
L'évolution du marché péruvien des produits laitiers depuis 1990 est également fortement liée au développement des voies de communication et des transports. Ce dernier conditionne en effet l'extension des circuits de collecte du lait des industries et le transport plus rapide jusqu'aux marchés urbains de produits périssables comme le lait cru, le fromage ou le yaourt. Certaines régions andines auparavant très isolées bénéficient d'un accès quotidien ou hebdomadaire en transport motorisé au marché qui devient dès lors porteur car accessible.

Le développement de la production laitière péruvienne au cours de la période s'explique ainsi par l'existence d'un marché attractif : prix élevés du fait de la protection aux frontières, expansion en volume sous l'effet de la croissance de la demande, accessibilité liée à l'amélioration des transports. Il faut cependant souligner les intérêts multiples de l'insertion de l'élevage laitier dans les systèmes de production ; la vente de produits laitiers permet l'obtention d'un revenu monétaire régulier, généralement utilisé pour couvrir les achats alimentaires nécessaires à la famille et complété par la vente occasionnelle de bovins sur pied lorsqu'un besoin de trésorerie se fait sentir. Dans la région étudiée, la spécialisation féminine du travail d'élevage autorise, en outre, la mise en place par les hommes d'activités non agricoles dont les revenus viennent compléter ceux issus de l'élevage. Se dessine ainsi une logique économique de sécurisation et de diversification des revenus autour du lait, qui contribue à en faire un produit porteur (3).

\section{Différenciation des systèmes de production laitiers péruviens}

Situés dans des écosystèmes contrastés, les systèmes de production qui approvisionnent les filières laitières péruviennes sont eux-mêmes très divers. Leur différenciation est liée à l'accès au foncier et à l'eau d'irrigation dont dépend le mode d'alimentation du troupeau bovin, mais aussi au capital qui conditionne la taille du cheptel et le choix d'une race plus ou moins productive.

Dans les Andes, la majeure partie des éleveurs bovins alimentent exclusivement leurs animaux au pâturage sur les parcours d'altitude et les résidus de culture après récolte (tableau I).

\section{Tableau I}

Typologie des systèmes de production laitiers andins *

\begin{tabular}{|c|c|c|c|c|c|c|}
\hline Mode d'alimentation & Race ou type & $\begin{array}{c}\text { Taille } \\
\text { troupeau }\end{array}$ & Main-d'œuvre & $\begin{array}{l}\text { Production laitière } \\
\text { (I/vache/j) }\end{array}$ & $\begin{array}{l}\text { Production laitière } \\
\text { (I/famille/j) }\end{array}$ & Zone \\
\hline $\begin{array}{l}\text { Parcours et } \\
\text { résidus de cultures }\end{array}$ & Criollo & 2-8 vaches & Familiale & $1-2$ & $2-15^{* *}$ & Huancavelica \\
\hline $\begin{array}{l}\text { Parcours et } \\
\text { résidus de cultures }\end{array}$ & Criollo & 9-30 vaches & Familiale & $1-2$ & $9-60 * *$ & Haut-Cañete \\
\hline $\begin{array}{l}\text { Cultures fourragères } \\
\text { irriguées et parcours }\end{array}$ & Criollo & $2-8$ vaches & Familiale & $3-6$ & $6-50$ & $\begin{array}{c}\text { Versant occidental, } \\
\text { Cajamarca (étage Jalca) }\end{array}$ \\
\hline $\begin{array}{l}\text { Cultures fourragères } \\
\text { en sec ou irriguées } \\
\text { et parcours }\end{array}$ & $\begin{array}{l}\text { Croisé criollo, } \\
\text { Brown Swiss } \\
\text { ou Holstein }\end{array}$ & $2-8$ vaches & Familiale & $5-10$ & $10-80$ & $\begin{array}{c}\text { Altiplano, Cajamarca } \\
\text { (versant) }\end{array}$ \\
\hline $\begin{array}{l}\text { Cultures fourragères } \\
\text { irriguées et concentrés }\end{array}$ & $\begin{array}{l}\text { Brown Swiss } \\
\text { ou Holstein }\end{array}$ & 2-8 vaches & Familiale & $8-25$ & $15-200$ & $\begin{array}{c}\text { Arequipa, Cajamarca } \\
\text { (vallée) }\end{array}$ \\
\hline $\begin{array}{l}\text { Cultures fourragères } \\
\text { irriguées et concentrés }\end{array}$ & $\begin{array}{l}\text { Brown Swiss } \\
\text { ou Holstein }\end{array}$ & 9-100 vaches & Salariée & $8-25$ & $70-2500$ & $\begin{array}{c}\text { Arequipa, Cajamarca } \\
\text { (vallée) }\end{array}$ \\
\hline
\end{tabular}

* Aubron, 2006

** En saison des pluies seulement 
Ils possèdent des troupeaux de 2 à 30 bovins de type criollo et ont une production de lait calée sur la saison des pluies, du fait du mode d'affouragement. L'extension de la période de traite à l'ensemble de l'année suppose que les éleveurs implantent des cultures fourragères, qu'ils irriguent ou stockent pour faire face à la saison sèche. Très présente dans les vallées interandines sèches et sur le versant occidental des Andes, la luzerne laisse place dans les Andes plus humides du Nord (Cajamarca) à l'association trèfle ray grass. Disposant de possibilités d'irrigation plus réduites, les éleveurs laitiers de l'Altiplano ou des environs de la ville de Huancavelica intensifient leur production laitière en semant de l'avoine qu'ils conservent sous forme de foin ou d'ensilage pour la saison sèche. Une distinction peut être faite entre les éleveurs qui exploitent, en plus de ces cultures fourragères, des surfaces de parcours à certaines périodes de l'année ou pour certains lots d'animaux, et ceux qui font de ces cultures fourragères le support presque exclusif de l'alimentation de leur troupeau, souvent complétée par la distribution de concentrés (tableau I). Ces derniers, jouissant d'une position géographique plus favorable et d'un accès plus large au foncier, élèvent généralement des animaux plus productifs, de race Holstein ou Brown Swiss, et non plus de type criollo, dont la rusticité perd de son intérêt dès lors qu'est abandonné le pâturage des parcours. Les animaux améliorés sont également très présents dans les élevages de la côte Pacifique qui sont pour la plupart en zéro pâturage. L'alimentation s'y fait à base de maïs, parfois ensilé, et de résidus de cultures irriguées (coton, canne à sucre, asperge) qui peuvent être produits sur l'exploitation ou achetés (6). La région côtière concentre également les élevages laitiers de type capitaliste, dans lesquels le travail de conduite de troupeaux de plusieurs centaines de têtes est effectué par des salariés.

Les volumes de lait produits quotidiennement par ces différents systèmes de production ne sont évidemment pas les mêmes (tableau I) : la production dans les petits élevages sur parcours est inférieure à 101 par jour par famille et en saison des pluies seulement ; elle est de quelques dizaines de litres par jour dans ceux combinant parcours et cultures fourragères, comme à Sinto, et atteint quelques centaines de litres par jour quand l'accès aux ressources permet l'élevage de plus de dix vaches plus productives, exclusivement nourries à partir de cultures fourragères et de concentrés. Enfin, les élevages de type capitaliste produisent quotidiennement plusieurs milliers de litres de lait.

La mise en concurrence de ces systèmes de production pour l'approvisionnement des différentes filières et les relations d'interdépendance entre filières se traduisent par une gamme de variation du prix du lait au producteur étroite par rapport à celle de ces volumes de production. Le prix du lait au producteur est de 50 centimes de soles par litre d'équivalent lait $(0,12 €)$ dans la partie périphérique des bassins d'approvisionnement des industries laitières et varie entre 60 et 90 centimes de soles $(0,14$ à $0,21 €)$ au cœur des bassins de collecte industrielle et dans les zones fromagères andines intégrées aux filières artisanales (11). Il atteint 1 sol par litre $(0,23 €)$ dans certains élevages de type capitaliste particulièrement prisés des industries du fait des économies d'échelle qu'ils autorisent et lorsqu'il est payé par l'Etat dans le cadre de programmes d'aide alimentaire de distribution de lait dans les écoles, par exemple. Ces variations de prix au producteur du simple au double sont donc bien loin de combler les écarts de volumes produits entre les différents types d'élevage, qui ne sont pas non plus proportionnels aux coûts monétaires qu'ils supportent. Il en résulte des écarts importants du niveau de productivité du travail et donc de revenus entre systèmes de production laitiers, ne serait-ce qu'entre les exploitations andines de type familial : un actif crée ainsi une valeur ajoutée annuelle d'environ 2000 soles (500 euros) avec un petit élevage de bovins criollos sur parcours, contre près de 40000 soles (10 000 euros) dans un système de production avec une dizaine d'hectares irrigués, autorisant l'élevage de 20 vaches laitières à partir de luzerne et de concentrés.

\section{Des filières artisanales marquées par l'essor des fromageries}

Dans de nombreuses régions andines, le développement de l'élevage laitier depuis 1990 s'accompagne de la mise en place de fromageries. Ces petites unités de transformation artisanale qui traitent quotidiennement entre 50 et 30001 de lait fonctionnent de manière associative ou privée et sont généralement informelles. Leur essor, qui n'est donc pas mesuré par les pouvoirs publics, est difficile à appréhender de façon chiffrée mais il a été attesté par les enquêtes dans toutes les régions laitières visitées, qu'il s'agisse de bassins laitiers également intégrés aux circuits de collecte industrielle (Cajamarca, Arequipa) ou plus isolés (Urubamba, Huancavelica, Puno). Ainsi, par exemple, jusqu'à huit fromageries, créées à partir de 1999, fonctionnaient en 2004 sur le territoire de la communauté étudiée.

Certaines de ces fromageries naissent de l'appui d'une institution de développement (ONG, programme public) qui joue parfois un rôle de relais entre les acteurs de la filière (30), là où d'autres, généralement moins bien équipées, sont le résultat d'initiatives individuelles. Leur essor s'explique à la fois par l'existence d'une demande urbaine pour le type de produit qu'elles élaborent et par l'alternative qu'elles représentent pour les ruraux dans un contexte de chômage urbain et de dégradation des revenus agricoles (4). L'objectif est à la fois d'accroître la valeur ajoutée par rapport à la filière fermière en se démarquant du fromage frais générique qu'elle élabore et de développer ses parts de marché face aux industries (8). A l'image de ce qui est observé dans les filières laitières d'autres régions du monde $(12,16,25)$, la souplesse d'organisation de la filière, l'origine territoriale, la typicité et la diversité des produits constituent autant de facteurs de compétitivité que la filière des fromageries naissante au Pérou cherche à mobiliser.

Ce dynamisme économique est bien à l'œuvre, que ce soit à travers le développement autour du queso mantecoso, dans la région de Cajamarca, d'un territoire fromager géré collectivement associant des activités de production, de transformation, de commercialisation et même de tourisme (9), ou autour des fromageries de Chuquibamba dans le sud du Pérou (région d'Arequipa) ; évinçant progressivement Gloria qui avait le monopole de la collecte du lait dans la zone il y a vingt ans, plusieurs fromageries familiales s'y sont créées. Elles élaborent un queso tilsit, dont la renommée va aujourd'hui jusqu'à Lima, qu'elles envisagent de commercialiser dans une chaîne de supermarché en adoptant un statut associatif (3). Situées au bord de la panaméricaine, la vingtaine d'unités de transformation artisanales du périmètre irrigué de Majes près d'Arequipa constituent un autre cas d'école : inexistantes sept ans plus tôt, elles collectent chacune, en 2005, 1000 à 50001 de lait par jour qu'elles transforment principalement en yaourt à boire, vendu aux passagers des bus qui s'arrêtent le long de la route. Plus de 100001 de ce nouveau produit sont ainsi écoulés quotidiennement (3). Enfin, les fromageries de Sinto collectent aujourd'hui près de la moitié des 20001 de lait produits quotidiennement dans la communauté et élaborent un fromage apprécié des consommateurs de classe moyenne des villes côtières et andines voisines (4).

L'analyse de ces expériences fait apparaître la plus forte mâ̂trise exercée par les fromageries sur l'aval de la filière par rapport à la filière fermière. La plupart des fromageries commercialisent au moins une partie de leurs produits à travers des circuits courts (réseaux familiaux, contrat oral de livraison régulière aux institutions, vente directe en bord de route, dans la rue ou au 
porte-à-porte), alors que les producteurs de fromage fermier vendent souvent à des commerçants, face auxquels ils ont un pouvoir de négociation restreint. Cependant, les fromageries ne permettent pas toujours d'accroître la valeur ajoutée créée par rapport à la filière fermière (figure 5) : dans certains cas, la différence de prix entre un produit fermier et un produit des fromageries ne couvre par le surcoût engendré par la collecte et la transformation. Les fromageries peinent alors à atteindre le seuil de rentabilité et le prix du lait au producteur est maintenu bas. Ainsi, dans la filière du queso mantecoso de Cajamarca, qui constitue un bon exemple du dynamisme de la filière des fromageries, le prix de l'équivalent lait payé aux producteurs de caillé reste l'un des plus faibles du pays $(11,19)$. Ces difficultés s'expliquent en partie par la forte concurrence à laquelle se livrent les fromageries pour l'approvisionnement des marchés urbains, qui se voit renforcée par l'absence de législation efficace concernant l'origine du produit et les techniques de transformation, et donc par la fréquence des fraudes.

\section{Diversification et nouvelle localisation des activités dans la filière industrielle}

Comme l'indiquait en 2005 le président de l'association des industries laitières : «Au Pérou, le lait évaporé en a encore pour un bon moment ». La diffusion de ce produit-phare des industries laitières auprès des classes populaires a été favorisée dans les années 1970 et 1980 par le contrôle exercé par l'Etat sur la filière laitière industrielle, relayé depuis le début des années 1990 par les programmes sociaux d'aide alimentaire et la publicité. La persistance de très fortes habitudes de consommation autour de ce produit laitier industriel bon marché n'exclut pas la mise en place de stratégies de diversification : depuis 1997, les industries péruviennes ont triplé leurs ventes de lait UHT, quadruplé celles de yaourt à boire, et doublé celles de fromage et de beurre (34). Cette diversification industrielle répond à l'évolution de la demande des consommateurs de classe moyenne et aisée qui, comme dans la plupart des villes latino-américaines, s'approvisionnent de plus en plus dans les supermarchés en plein développement (31). Elle s'explique aussi par la rupture politique des années 1990, puisque les entreprises laitières, qui transformaient des matières premières laitières subventionnées en un produit standard écoulé sur un marché contrôlé par l'Etat, affrontent aujourd'hui une concurrence accrue. Ainsi, dans la première moitié des années 1990, le lait en poudre en sachet Anchor, importé et commercialisé par l'entreprise néozélandaise The New Zealand Dairy Board pour la consommation directe, menaçait-il de détrôner la boîte de lait évaporé (5). Visible à travers les prix au consommateur (figure 5), la stratégie de diversification des industries peut se résumer ainsi : il s'agit d'écouler auprès des classes sociales à hauts revenus des produits à forte

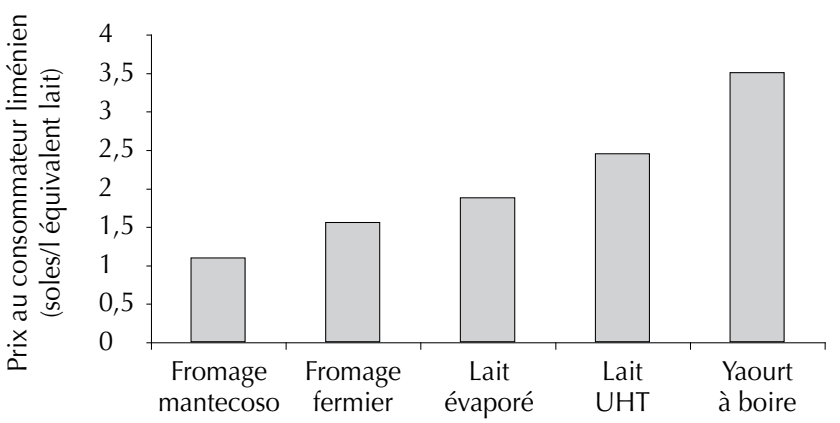

Figure 5 : la transformation ne génère pas toujours de valeur ajoutée ; prix au consommateur de cinq produits laitiers, mai 2005 (équivalents lait rendements). Grézes, 2000 ; Inei, 2005. valeur ajoutée (lait UHT, yaourt) tout en conservant les parts de marché auprès des classes populaires, avec de gros volumes de vente de lait évaporé.

L'évolution du contexte conduit également les industries à repenser la localisation de leurs activités, à l'échelle nationale, continentale, voire mondiale. Au Pérou, la tendance est à la concentration des activités de transformation à proximité de la capitale et à l'expansion des circuits de collecte vers de nouveaux bassins d'approvisionnement. Les régions côtières (Lima, La Libertad, Ica), dans lesquelles la production laitière stagnait dans les années 1980, connaissent un certain renouveau. Les accords de libre-échange, qui tendent à réduire le prix du maïs et du soja importés, y sont favorables aux exploitations de type capitaliste, qui attirent les industries du fait des volumes qu'elles produisent, et donc des économies d'échelle qu'elles autorisent. La compétitivité de ces élevages côtiers est néanmoins étroitement dépendante des prix relatifs des produits et des intrants, ce qui conduit les industries à maintenir et développer leurs activités de collecte dans d'autres parties du Pérou. Elles s'installent dans les régions les plus accessibles et où la productivité du travail est susceptible de s'accrô̂tre. Le piémont amazonien présente à ce titre un potentiel important que l'entreprise Gloria projette de mobiliser dans les années à venir : la construction de routes y rend la collecte moins coûteuse et le lait, sous-produit de l'élevage bovin allaitant, peut être acheté à bas prix.

De manière analogue à l'échelle continentale, les industries laitières raisonnent leur installation dans chaque région pour y développer des activités de collecte, de transformation ou de commercialisation en fonction des avantages propres à chaque situation : productivité élevée du travail et donc faible coût de la matière première, présence d'usines de transformation performantes, fort potentiel de croissance de la consommation, etc. C'est ainsi que l'entreprise péruvienne Gloria qui domine le marché national des produits laitiers industriels contrôle également la totalité de la production bolivienne de lait en poudre, importée au Pérou sans taxe depuis 1997, en vertu des accords la Communauté andine (CAN). Au Pérou, Gloria mélange le lait en poudre importé au lait liquide national et élabore ainsi du lait évaporé, dont elle exporte, depuis 1999, une partie croissante vers Haïti et plusieurs pays d'Afrique de l'Ouest. En 2005, l'entreprise péruvienne s'est également établie en Colombie, puis en Argentine où elle a acheté une usine de fabrication de lait en poudre d'une capacité d'un million de litres de lait par jour et dont la production pourra être importée sans droit de douane au Pérou si celui-ci intègre le Marché commun austral (Mercosur).

\section{DISCUSSION}

\section{Production laitière péruvienne : un développement inégal}

Répondant à l'existence d'un marché national protégé des importations, en expansion du fait de la croissance démographique urbaine et rendu accessible par l'amélioration du réseau routier, la production laitière péruvienne présente depuis 1993 un taux de croissance annuel de l'ordre de 5 p. 100. Le développement résultant des filières laitières semble concerner les filières artisanales comme la filière industrielle, mais ils se traduit par de fortes inégalités de revenus entre producteurs. Reflet d'un accès différencié aux ressources foncières et hydriques pour la production fourragère, l'écart de productivité du travail entre les systèmes de production laitiers des Andes est en effet d'un facteur 20. Les grandes étables laitières de la côte Pacifique, dont la productivité varie en fonction des rapports de prix, bénéficient actuellement d'une conjoncture 
plutôt favorable et correspondent aux systèmes de production les plus compétitifs du pays (7). Loin derrière elles, les systèmes de production de Sinto, pourtant relativement spécialisés dans l'élevage bovin, permettent difficilement d'obtenir 1000 euros de revenu par famille et par an à partir d'un hectare irrigué.

Le même contraste caractérise la consommation péruvienne de produits laitiers : la croissance de la production laitière alimente l'expansion de la demande en volume mais la consommation moyenne par habitant stagne depuis dix ans ; elle reste nulle ou très faible pour la frange la plus pauvre de la population et se rapproche de celle des pays développés pour les catégories sociales aisées, qui ont un plus large éventail de choix qu'auparavant dans l'étalage des supermarchés. Si le marché péruvien des produits laitiers est bel et bien un marché porteur depuis 1990, c'est donc au prix de fortes inégalités de développement que seule la mise en place d'une politique adaptée peut permettre de résoudre.

\section{Enjeux et limites des démarches qualité dans les filières fromagères artisanales}

Dans ces conditions peu favorables aux petits éleveurs laitiers, l'intégration dans une filière fromagère artisanale permettant d'accrồtre la création de valeur ajoutée et de faire évoluer sa répartition au bénéfice des producteurs apparaît comme une solution. Cette stratégie, appuyée par certains organismes de développement, passe par la mise en place de démarches qualité combinant des éléments aussi divers que l'amélioration de la qualité sanitaire, visuelle et gustative des produits, la revendication de leur origine territoriale, la caractérisation de leur typicité ou la commercialisation dans des circuits courts reposant sur des relations de confiance entre producteurs et consommateurs.

Entreprises avec succès dans certaines situations locales préalablement décrites, ces démarches qualité n'en restent pas moins une solution fragile et difficilement généralisable à l'ensemble de la production fromagère andine. Le pouvoir d'achat limité de la population péruvienne constitue l'une des principales limites à leur développement. L'essentiel des fromages artisanaux est écoulé aujourd'hui à bas prix sur les marchés populaires et dans les bidonvilles de Lima par le biais de réseaux familiaux ; le marché péruvien des produits laitiers n'a pas, dans le contexte actuel, la capacité d'absorber de tels volumes de fromage de qualité, parce que la majorité de la population péruvienne est pauvre et n'a pas les moyens de les acquérir. De plus, dans le modèle de consommation adopté par les classes moyennes et aisées de la côte qui s'approvisionnent de plus en plus dans les supermarchés, les produits laitiers artisanaux sont mal représentés et concurrencés par leurs équivalents industriels. Ainsi, en dépit des démarches qualité mises en place dans certaines filières, les fromages artisanaux constituent en moyenne, d'après les données de l'Inei, les produits laitiers les moins chers du marché (figure 5). Loin d'ajouter de la valeur au lait, la transformation fromagère artisanale n'est donc bien souvent qu'un moyen de le conserver et de le transporter jusqu'aux marchés urbains, sur lesquels il est vendu à bas prix auprès d'une population pauvre.

\section{Industries et filières artisanales dans la libéralisation des échanges}

D'après la base de données des douanes, les pays avec lesquels le Pérou a engagé des négociations commerciales visant la suppression des barrières douanières y exportent à l'heure actuelle peu de produits laitiers. Mais à l'image de ce qui s'est passé avec la Bolivie, qui a vu ses exportations laitières vers le Pérou s'accroître suite à la création de la CAN, les accords de libre-échange peuvent modifier la structure des importations. Tout dépend de la compétitivité des producteurs et des transformateurs de lait des pays étrangers sur le marché péruvien, accrue par la suppression des taxes à l'importation. Or, les producteurs nord-américains et colombiens sont, pour des raisons différentes, compétitifs face aux éleveurs laitiers péruviens. Ceci est encore plus vrai des élevages argentins qui reposent sur le pâturage d'immenses surfaces de parcours par des troupeaux de plusieurs centaines de têtes et qui figurent parmi les plus compétitifs du monde. Pour ces pays, qui sont de plus autosuffisants en produits laitiers, la suppression des barrières douanières avec le Pérou constitue donc, à des degrés divers, une opportunité commerciale.

Avec l'ouverture des frontières, la concurrence des produits laitiers importés sur le marché péruvien est ainsi amenée à s'accroître. Elle concerne le marché de certains produits finis (lait en poudre en sachet contre lait évaporé, fromages) mais également le marché de la matière première laitière. A l'image de la situation des années 1970 et 1980 (24) ou de celle qui prévaut aujourd'hui au Mexique (27), le lait en poudre importé peut, s'il est moins cher, remplacer une partie du lait national collecté par les industries dans la composition du lait évaporé. Qu'elle concerne les produits finis ou la matière première, cette concurrence accrue des importations exerce une pression à la baisse sur le prix du lait au Pérou que les industries répercutent à leurs fournisseurs. Dans ce nouveau contexte, les filières artisanales sont certes avantagées par le fait qu'elles élaborent des produits lourds et périssables (yaourt, fromage) moins concurrencés par les importations. Mais, elles ne sont pas seules sur ces segments de marché puisque les industries entreprennent aussi une diversification de leur production. Par ailleurs, du fait de l'interdépendance entre filières laitières, les éleveurs approvisionnant les filières artisanales ne sont pas épargnés par la baisse du prix de l'équivalent lait au producteur imposée par les industries.

\section{CONCLUSION}

Cette analyse invite à la mise en place d'une politique laitière dans ce pays andin, dont le but serait de réduire les inégalités actuellement générées en faisant de la production et des filières laitières un véritable moteur de développement. Le premier volet d'une telle politique pourrait être axé sur la mise en place d'un appui technique adapté aux petits et moyens producteurs et constituer le cœur d'une politique de l'élevage pour les Andes péruviennes. La recherche et la diffusion de variétés fourragères plus résistantes aux conditions climatiques andines, le développement de techniques peu onéreuses de stockage fourrager pour la saison sèche, d'infrastructures et de modalités d'irrigation plus économes en eau, ou la sélection d'animaux rustiques mais à meilleur potentiel laitier constituent autant de pistes de travail aujourd'hui peu investies par les institutions publiques. Elles permettraient pourtant d'accroître la productivité du travail dans ces unités de production qui sont nombreuses et pour certaines en situation de grande pauvreté.

L'appui aux filières artisanales constituerait le deuxième volet de cette politique laitière péruvienne. Le maintien de la taxe à l'importation des produits laitiers est nécessaire pour maintenir le prix intérieur, mais insuffisant pour garantir le développement des filières artisanales compte tenu des écarts de productivité entre producteurs nationaux. Une segmentation plus marquée depuis l'amont entre les sous-filières laitières permettrait de faire coexister plusieurs niveaux de prix mieux en rapport avec la productivité du travail. Il faudrait pour cela que l'Etat, en s'inspirant d'expériences de démarche qualité réussies, identifie et délimite les filières artisanales avec les acteurs concernés et institue un système de 
sanction des fraudes. L'Etat a également un rôle à jouer en aval de la filière dans la promotion de la consommation des produits laitiers artisanaux, en favorisant leur accès au marché par la construction d'infrastructures ou en les intégrant aux programmes d'aide alimentaire, aujourd'hui dominés par les produits industriels. Resterait la limite imposée par la faiblesse du pouvoir d'achat de la population péruvienne. Rappelons à ce titre que l'amélioration des revenus des petits et moyens producteurs constitue un moyen pour le Pérou d'élargir son marché intérieur. A Sinto, bon nombre d'éleveurs n'ont en effet jamais goûté le fromage élaboré par les fromageries auxquelles ils livrent leur lait, faute de moyens pour les acquérir.

\section{BIBLIOGRAPHIE}

1. ABLAN E., 1989. Les produits andins au Pérou. Thèse Doct., Iheal, Paris III, France, 387 p.

2. AMAT Y LEON C., 1983. Niveles de vida y grupos sociales en el Perú Lima, Perú, Universidad del Pacífico, 249 p.

3. AUBRON C., 2006. Le lait des Andes vaut-il de I'or ? Logiques paysannes et insertion marchande de la production fromagère andine. Thèse Doct., INA-P-G, Paris, France, 480 p.

4. AUBRON C., MOITY-MAIZI P., 2007. Des fromageries dans un territoire andin marginal : une innovation pour le développement local ? Econ. Soc., Série AG, 29 : 1525-1546.

5. BERNET T., 1998. Desarrollo del sector lácteo peruano: pasado y presente, documento base para investigaciones futuras. Lima, Perú, CIP, $47 \mathrm{p}$.

6. BERNET T., JULCA J., SAENZ J., PRAIN G., 2000. Peri-urban milk production in Peru: Assessing farmers' decision-making within a changing market. Livest. Res, rural Dev., 12: 81-100.

7. BERNET T., STAAL S., WALKER T.S., 2001. Changing milk production trends in Peru: Small-scale highland farming versus coastal agrobusiness. Mt. Res. Dev., 21: 268-275.

8. BOUCHER F., 2004. Enjeux et difficultés d'une stratégie collective d'activation des concentrations d'agro-industries rurales, le cas des fromageries de Cajamarca, au Pérou. Thèse Doct., université de Versailles / St Quentin en Yvelines, France, 434 p.

9. BOUCHER F., CARIMENTRAND A., REQUIER-DESJARDINS D. 2003. Agro-industries rurales et lutte contre la pauvreté. Les systèmes agroalimentaires localisés contribuent-ils au renforcement des "capabilités»? In : Actes atelier, Montpellier, France, 11-12 sept. 2003 Montpellier, France, Cirad, 20 p.

10. BRAUN E., STEINDL J., 2004. Gloria S.A. Lima, Perú, Apoyo y asociados, $15 \mathrm{p}$.

11. CEPES, 2003, 2004, 2005, 2006. Boletín vida láctea $n^{\circ} 11-27$.

12. CERDAN C., SAUTIER D., 2001. Réseau localisé d'entreprises et dynamique territoriale. Le bassin laitier de Gloria (Nordeste Brésil). Etud. Rech. Syst. agraires Dév., 32 : 131-144.

13. COCHET H., 2005. L'agriculture comparée. Genèse et formalisation d'une discipline. Paris, France, INA P-G, 88 p.

14. DEL VALLE RIVERA M.C., ALVAREZ A., 1998. Les vicissitudes de la production laitière dans le Fraylesca, Chiapas. In : Bey M., Ed., Politiques néolibérales et acteurs ruraux au Mexique. Paris, France, L'Harmattan, p. 211-233.

15. DUFUMIER M., 2002. Systèmes d'élevage laitier dans le monde évolutions récentes et nouveaux enjeux. In : $26^{\mathrm{e}}$ Congrès mondial de laiterie, Paris, France, 24-27 sept. 2002, 12 p

16. DUTEURTRE G., 1998. Compétitivité prix et hors-prix sur le marché des produits laitiers $d^{\prime}$ Addis-Abeba (Ethiopie). La production fermière face à ses nouveaux concurrents. Thèse Doct., Montpellier, France, Ensam, Cirad, $353 \mathrm{p}$

17. FRANQUEVILLE A. 1993. Surproduction et pénurie de lait en Bolivie. Quand la libéralisation désorganise la production nationale. Cah. Sci. Hum., 29 : 139-151.

18. GHERSI G., BENCHARIF H., 1992. De I'agriculture à l'aliment : les opérations et les composantes du SAA. In : Malassis L., Ghersi G., Eds, Initiation à I'économie agro-alimentaire. Paris, France, Uref, p. 94-108.

19. GREZES J.P., 2000. La production fromagère à Cajamarca (Pérou) : un exemple de "système agro-alimentaire localisé ». Mémoire fin études, Montpellier, France, Cnearc, 113 p.

20. INEI, 2001. Perú: Compendio estadístico 2001. Lima, Perú, INEI.

21. INEI, 2003. Perú: Compendio estadístico 2003. Lima, Perú, INEI.

22. INEI, 2004. Indicadores de precios de la economía. Lima, Perú, INEI.

23. INEI, 2005. Precios promedios mensuales de productos lácteos seleccionados del índice de precios al consumidor de Lima metropolitana, enero 1980 - mayo 2005. Lima, Perú, INEI, 6 p.

24. LAJO LAZO M., 1988. Dependencia alimentaria y reactivación de la crísis, Perú, 1970 - 1985 - 1988. Lima, Perú, CENES, 273 p.

25. LINCK T., 2005. Patrimonialisation et typification de fromages traditionnels : une approche comparée de démarches de qualification. In : Colloq. SFER Au nom de la qualité. Quelle(s) qualité(s) demain, pour quelle(s) demande(s) ? Clermont-ferrand, France, 15-16 déc. 2005

26. MALASSIS L., 1979. Economie agro-alimentaire, tome I. Economie de la consommation et de la production agro-alimentaire. Paris, France, Cujas, $437 \mathrm{p}$.

27. MARTineZ E., ALVAREZ A., GARCiA L.A., DEL VAlLE M.C., 1999. Dinámica del sistema lechero mexicano en el marco regional y global. México, México, Plaza y Valdés, 412 p.

28. MEYER C., DUTEURTRE G., 1998. Equivalents lait et rendements en produits laitiers : modes de calcul et utilisation. Revue Elev. Méd. vét. Pays trop., $51: 247-257$.

29. MINAG, 2003. Estadística agraria mensual, dic. 2003. Lima, Perú, MINAG, $169 \mathrm{p}$.

30. REQUIER-DESJARDINS D., 1999. Agro-industria rural y sistemas agroalimentarios localizados $i$ Cuales puestas ? In : $X$ aniversario de PRODAR, Quito, Ecuador.

31. REARDON T., BERDEGUE J., 2002. The rapid rise of supermarkets in Latin America: Challenges and opportunities for development. Dev. Policy Rev., 20: 371-388.

32. ROMAN C., 1986. Politiques et stratégies paysannes d'élevage bovin au Pérou. Le cas de Cañete et Yauyos. Mémoire Dess, ledes, Paris, France, $226 \mathrm{p}$.

33. VALCESCHINI E., 1995. Contrat, coordination et institutions. Problématiques et méthodologies de l'économie rurale. In : Allaire G., Boyer R., Eds., La grande transformation de I'agriculture. Paris, France, Inra Economica, p. 241-258

34. WEBB R., FERNANDEZ BACA G., 2004. Perú en Números. Lima, Perú, Cuánto, 1178 p.

Reçu le 15.05.2007, accepté le 01.04.2008 


\section{Summary}

Aubron C. Milk and Cheese in an Andean Country: What Place for the Peruvian Traditional Subsector Faced with Dairy Industries?

Since the beginning of the 1990s, Peruvian milk production has increased greatly. The development of dairy supply chains is linked to the growth of a market protected from importations, and which is expanding because of urban population growth and improvements of the road network. It concerns both small dairy producers and the industrial dairy subsector, which are connected by interdependent and balance of power relations all along the chain. Dairy farmers were surveyed from a technical and economic angle in various regions. Results show that this dairy development brings about major income inequalities among producer types, reflecting an unequal access to resources. Statistical data in the literature and interviews of actors of the Peruvian dairy chains allow to assess the stakes and limits of quality approaches in the small producers' chain faced with industries. Finally, the article questions the impact of free-trade agreements in which Peru is involved with regard to the domestic dairy subsector, and concludes with political proposals to accompany dairy development.

Keywords: Animal husbandry - Milk - Cheese - Informal sector - Dairy industry - Agricultural policy - Andean region Peru.

\section{Resumen}

Aubron C. ¿Leche y queso en un país andino: cual es el lugar de las cadenas artesanales peruanas frente a las industrias lecheras?

Desde el inicio de los años 1990, la producción de leche peruana se encuentra en fuerte crecimiento. El desarrollo de cadenas lecheras responde al de un mercado protegido de las importaciones, en expansión debido al crecimiento demográfico urbano y ahora accesible gracias a la mejora de la red de carreteras. Concierne las cadenas artesanales como industriales, ligadas de principio a fin por relaciones de interdependencia y de equilibrio de poder. A partir de encuestas técnico-económicas de los productores de leche en diferentes regiones, el presente artículo muestra que este desarrollo lechero se traduce por importantes desigualdades de ingresos entre las categorías de productores, reflejo de un acceso diferente a los recursos. Los datos estadísticos consultados y las entrevistas Ilevadas a cabo con los diferentes participantes de las cadenas peruanas permiten la evaluación de los argumentos en juego y de los límites de las medidas de calidad puestas en aplicación en las cadenas artesanales para afrontar a las industriales. Finalmente, el artículo se interroga sobre el impacto de los acuerdos de libre comercio, en los cuales Perú se compromete sobre las cadenas lecheras nacionales y concluye con propuestas de políticas de acompañamiento de este desarrollo lechero.

Palabras clave: Ganadería - Leche - Queso - Sector informal Industria lechera - Politica agrícola - Región andina - Perú. 